\title{
Critical nucleotides in the interaction of CatR with the pheBA promoter: conservation of the CatR-mediated regulation mechanisms between the pheBA and catBCA operons
}

\author{
Andres Tover, ${ }^{1}$ Jana Zernant, ${ }^{1}$ Sudha A. Chugani, ${ }^{2} \dagger$ \\ Ananda M. Chakrabarty ${ }^{2}$ and Maia Kivisaar ${ }^{1}$
}

Author for correspondence: Maia Kivisaar. Tel: +372 7375 015. Fax: +372 7420286. e-mail:maiak@ebc.ee

1 Department of Genetics, Institute of Molecular and Cell Biology, Estonian Biocentre and Tartu University, 51010 Tartu, Estonia

2 Department of Microbiology and Immunology, University of Illinois College of Medicine, Chicago, Illinois, USA

\begin{abstract}
The promoter of the plasmid-borne pheBA genes encoding enzymes for phenol degradation resembles the catBCA promoter and is activated by CatR, the regulator of the chromosomally encoded catechol-degradative catBCA genes in Pseudomonas putida. In this study, site-directed mutagenesis of the pheBA promoter region was performed. The interrupted inverted repeat sequence of the CatR recognition binding site (RBS) of the pheBA promoter is highly homologous to that of the catBCA promoter. However, the RBS was shown not to be the sole important feature for high-affinity binding of CatR to this site. Mutagenesis of the activation binding site (ABS) of CatR, which overlaps the -35 hexamer sequence TTGGAT of the promoter, revealed that the two G nucleotides in this sequence are important for promoter activity but not for CatR binding. All other substitutions made in the ABS negatively affected both the promoter activity and CatR binding. The spacer sequence of the pheBA and catBCA promoters between the -10 and -35 hexamers is $19 \mathrm{bp}$, which is longer than optimal. However, reducing the spacer region of the pheBA promoter was not sufficient for CatR-independent promoter activation. An internal binding site (IBS) for CatR is located downstream of the transcriptional start site of the catBCA genes and it negatively regulates the operon. A similar IBS was identified in the case of the pheBA operon and tested for its functionality. The results indicate a conservation of CatR-mediated regulation mechanisms between the pheBA promoter and the catBCA promoter. This universal mechanism of CatR-mediated transcriptional activation could be of great importance in enabling catechol-degrading bacteria to expand their substrate range via horizontal transfer of the phenol degradative genes.
\end{abstract}

Keywords: Pseudomonas putida, transcription activation, CatR, pheBA and catBCA operons, evolution of catabolic pathways

\section{INTRODUCTION}

A comparison of the major pathways for catabolism of aromatic compounds in bacteria has revealed that these compounds are transformed by different enzymes to a limited number of central intermediates, such as proto-

\footnotetext{
† Present address: Department of Microbiology, University of lowa, lowa City, IA 52242, USA.

Abbreviations: $\mathrm{ABS}$, activation binding site; $\mathrm{CCM}$, cis,cis-muconate; $\beta$ Gal, $\beta$-galactosidase; IBS, internal binding site; RBS, recognition binding site.
}

catechuate and (substituted) catechols (Harayama \& Timmis, 1989). In Pseudomonas species, unsubstituted phenols and haloaromatic compounds like chlorocatechols are usually metabolized by an ortho-cleavage pathway in which catechol is cleaved by catechol 1,2dioxygenase. The cleavage product cis,cis-muconate (CCM) is transformed via $\beta$-ketoadipate-enol-lactone to succinate and acetyl coenzyme A (Ornston, 1966). Genes encoding catechol degradation enzymes (cat genes) are located on the chromosome of Pseudomonas putida (Aldrich \& Chakrabarty, 1988). The genes of the modified ortho-cleavage pathways, including clcABD 
(a)

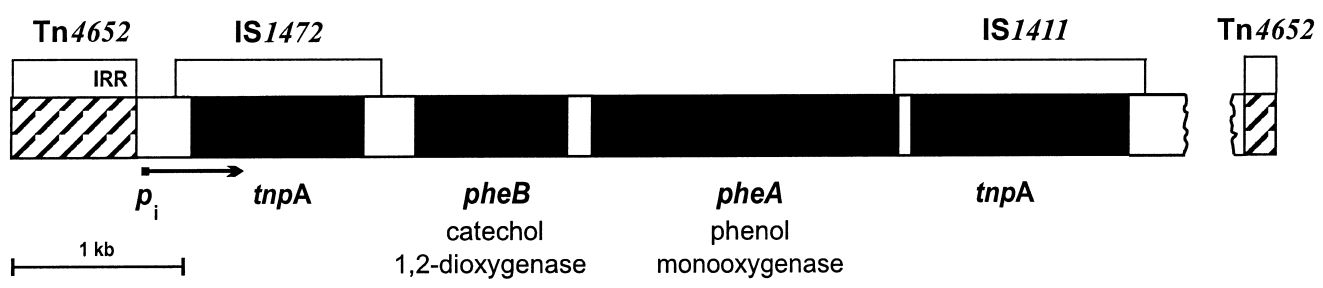

(b)
RBS
$\mathrm{ABS}$
pheBA

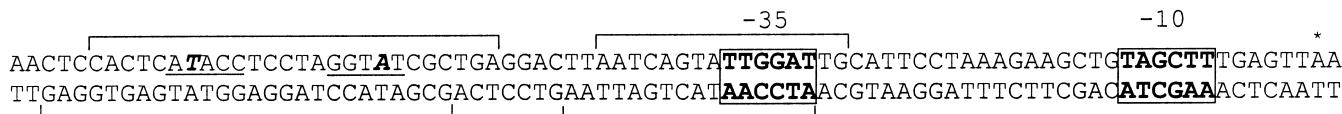

RBS

ABS

catBCA

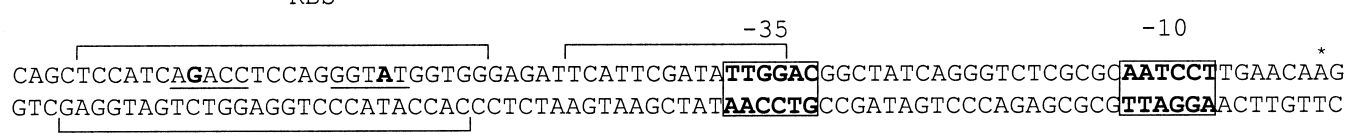

(c)

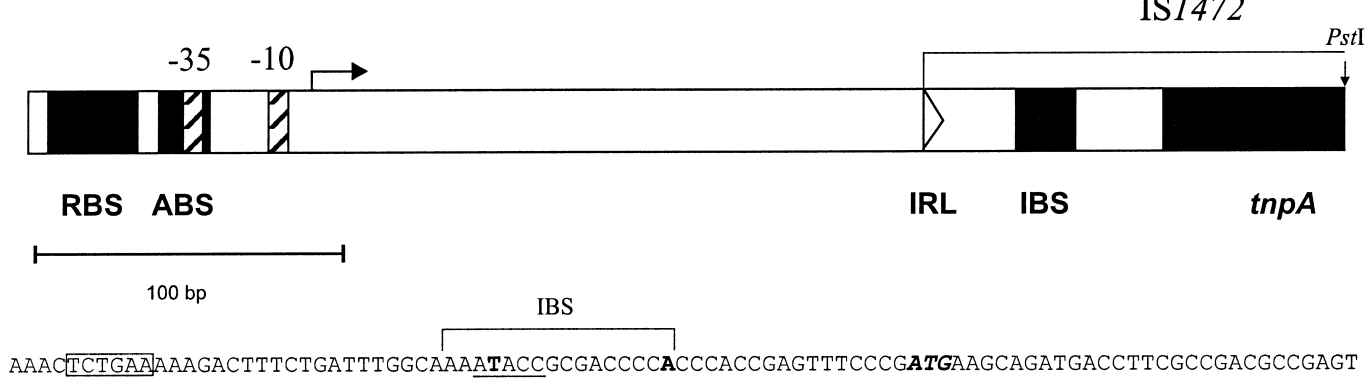

IRL (IS 1472)

$\operatorname{tnp} A$

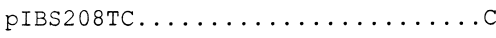

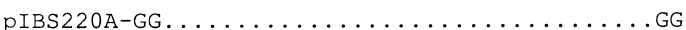

Fig. 1. (a) Organization of the pheBA operon on plasmid pAT1140 (Kasak et al., 1993). The genes phe $B$ and pheA are flanked by two IS elements, IS1472 and IS1411 (Kallastu et al., 1998). The right-end sequences of the transposon Tn4652 (Tsuda et al., 1989) are shown by hatched boxes. IRR indicates the $46 \mathrm{bp}$ terminal inverted repeat of Tn 4652 . Black boxes show the locations of the pheBA genes and the transposase genes (tnpA) of IS1472 and IS1411. The open boxes represent the intergenic region. The promoter of the pheBA operon (designated $p_{\mathrm{i}}$ ) is located upstream of IS1472. The arrow indicates the direction of transcription of the genes. (b) Nucleotide sequences of the pheBA and catBCA promoter regions. The bracketed sequences represent nucleotides protected by CatR from cleavage by DNase I (Parsek et al., 1995; Chugani et al., 1997). The protected regions are designated as RBS and ABS. The nucleotides of the interrupted inverted repeat within the RBS are underlined. The boldface italic represents the T and A residues of the conserved T-N $\mathrm{N}_{11}-\mathrm{A}$ of the LysR family regulatory proteins binding motif present in the RBS (Goethals et al., 1992). The catBCA promoter has a G instead of the conserved T. The nucleotides of the -35 and -10 elements of the promoters are in boldface and boxed. The transcriptional start sites are denoted by an asterisk. (c) The location of the CatR IBS in the pheBA operon. The CatR binding sites RBS, ABS and IBS are indicated by black boxes. The -35 and -10 hexamers of the pheBA promoter are marked by hatched boxes. The sequence of IBS is located in IS1472, between the left inverted repeat (indicated by a triangle) and the transposase gene tnpA (shown in black) of the element. The nucleotide sequence of the IBS region of the pheBA operon is shown below. The location of the nucleotides belonging to the IBS (indicated by a bracket) was originally proposed by Parsek et al. (1996). The sequence identical to one half of the interrupted inverted repeat of the RBS is underlined and the $T$ and $A$ residues of the conserved $T-N_{11}-A$ of the LysR binding motif are in bold. The left inverted repeat of $I S 1472$ is boxed and the putative translation initiator codon ATG of the tnpA gene is in boldface italics. 
(Franz \& Chakrabarty, 1987) and tcbCDEF (van der Meer et al., 1991), have been characterized in bacteria capable of degrading chlorocatechols and are located on catabolic plasmids. Transcription of the cat $B C A$, $c l c A B D$ and $t c b C D E F$ operons is controlled by the regulatory proteins CatR (Rothmel et al., 1990), ClcR (Coco et al., 1993) and TcbR (van der Meer et al., 1991), respectively. These activators belong to the LysR family of bacterial regulators and utilize similar mechanisms for activating transcription (McFall et al., 1997, 1998).

Many micro-organisms metabolize the toxic compound phenol via catechol as an intermediate. The catechol derived from phenol is usually metabolized by a different pathway known as the meta pathway (Herrmann et al., 1988; Kukor \& Olsen, 1990; Shingler et al., 1992; Wigmore et al., 1977). The pheBA genes specifying phenol degradation originate from multiplasmid Pseudomonas sp. strain EST1001 (Kivisaar et al., 1990). phe A encodes phenol monooxygenase, which converts phenol to catechol, while pheB encodes catechol 1,2dioxygenase. When the plasmid-borne pheBA operon (Fig. 1a) is introduced into a $P$. putida strain which is normally incapable of utilizing phenol, the bacteria acquire the ability to degrade phenol via the chromosomal cat pathway encoding catechol degradation in concert with the phenol to catechol or CCM conversion mediated by the pheBA operon (Kasak et al., 1993). Transcription of the chromosomal ortho pathway genes catBCA and of the plasmid-encoded pheBA operon is activated by the same positive regulator, the chromosomally encoded LysR family protein CatR (Kasak et al., 1993; Rothmel et al., 1990). Despite the different origins of the catBCA and the pheBA promoters, the CatR-dependent transcriptional activation mechanism of these promoters seems to be similar. This is therefore an example of how evolution took a short-cut to simply evolve a couple of genes for phenol degradation, instead of a whole pathway with its cluster of structural and regulatory genes that normally evolves in microorganisms.

A comparison of the nucleotide residues in the pheBA and catBCA promoters suggests that the two promoters are highly conserved and the DNase I protection patterns of CatR on these two promoter sequences are very similar (Chugani et al., 1997; Parsek et al., 1995) (Fig. $1 b)$. CatR binds to the pheBA and the cat $B C A$ promoters in both the presence and absence of the inducer but activates transcription only in the presence of the inducer CCM (Parsek et al., 1995; Rothmel et al., 1991). In the absence of CCM, CatR binds to the recognition binding site (RBS). CCM allows CatR to bind to a second site, the activation binding site (ABS) and to activate transcription from the promoter. Binding of CatR to the $A B S$ of the catBCA or the pheBA promoter is cooperative, requiring the presence of an intact RBS (Kasak et al., 1993; Parsek et al., 1992, 1995). Footprinting studies revealed that the ABS overlaps the -35 hexamer of the promoters (Chugani et al., 1997; Parsek et al., 1992, 1995). The C-terminal domain of the $\alpha$-subunit of RNA polymerase is required for the expression of the cat $B C A$ and the pheBA operons because RNA polymerases with truncated $\alpha$-subunits were deficient in activation (Chugani et al., 1997). Recently, an internal binding site (IBS) for CatR was identified within the catB structural gene. The IBS negatively regulates the expression of the cat $B C A$ promoter and the repression is CatR-mediated (Chugani et al., 1998).

To study how similar or dissimilar the pheBA promoter region is to the catBCA promoter region, not just in terms of DNA homology but also in terms of functionality, we conducted site-directed mutagenesis in the RBS, the ABS and the putative IBS of the pheBA promoter. Comparing the effect of these mutations with those introduced into the catBCA promoter region (Parsek et al., 1994) allowed us to determine critical nucleotides involved in direct interactions with CatR at both promoters. Data obtained in this study demonstrate that although some interactions of CatR with the RBS seem to be different between these two promoters, both the promoters utilize a very similar mechanism for transcriptional activation.

\section{METHODS}

Bacterial strains, plasmids and media. Escherichia coli TG1 (Carter et al., 1985) was used for the DNA cloning procedures and P. putida PaW85 (Bayley et al., 1977) was used as the host strain to study the expression of the pheBA promoter mutants. E. coli BL21(DE3) (Studier \& Moffat, 1986) was used for inducible overexpression of CatR in the pET11 vector under the control of the T7 promoter. The DNA fragment containing the cat $R$ gene was subcloned as a $\sim 1 \mathrm{~kb} H$ indIII-EcoRI fragment into pBluescript $\mathrm{SK}(+)$. To obtain the CatRoverproducing plasmid pET11CatR, the catR sequence was amplified with primers CatRNde 5'-AAG(NdeI) CATATG GAGCTGCGCCACTTGCGT-3' (complementary to the pET11 sequences containing the NdeI site and to the first seven codons for CatR) and the Reverse primer 5'-AACAGCTATGACCAG-3' [complementary to pBluescript $\mathrm{SK}(+)$ sequences flanking the multicloning sites in this plasmid]. The 950 bp PCR fragment was cleaved with NdeI and BamHI [the $\mathrm{BamHI}$ site in the amplified DNA region was derived from pBluescript $\mathrm{SK}(+)$ multicloning sequence] and inserted into the NdeI- and BamHI-cleaved protein expression plasmid pET11. The $158 \mathrm{bp}$ DNA fragment containing the pheBA promoter region was amplified by PCR using the primers catRylem (5'-TCCGAATTC $(E c o R I)$ TTGCCTTATCTCGGCATAACCC-3') and catRall (5'- GGCATCGAT(ClaI)TGCCTCCCAACTTTTAGTCTTG-3'), complementary to nucleotides -132 to -103 and +14 to +45 , respectively, relative to the transcriptional start site of the pheBA promoter. The PCR-generated DNA fragment, cloned into pBluescript $\mathrm{SK}(+)$, was inserted with BamHI and XhoI ends into the promoter-probe vector pKTlacZ (Hõrak \& Kivisaar, 1998). The 795 bp DNA fragment carrying the pheBA promoter region with the CatR IBS was cloned as a PstI-Eco47III DNA fragment into pBluescript $\mathrm{SK}(+)$ and then, using the BamHI and $\mathrm{XhoI}$ ends, into pKTlacZ.

Bacteria were grown on Luria-Bertani (LB) medium (Miller, 1992) or on M9 minimal medium (Adams, 1959). Antibiotics were added at the following final concentrations: for E. coli, ampicillin $100 \mu \mathrm{g} \mathrm{ml}^{-1}$; and for $P$. putida, carbenicillin at $1500 \mu \mathrm{g} \mathrm{ml} l^{-1}$. P. putida was incubated at $30^{\circ} \mathrm{C}$ and E. coli at 
$37^{\circ}$ C. E. coli was transformed with plasmid DNA as described by Hanahan (1983) and DNA was electroporated into $P$. putida by the protocol of Sharma \& Schimke (1996).

Site-directed mutagenesis of the pheBA promoter region and DNA sequencing. Site-directed mutagenesis of the pheBA promoter region and the IBS sequence was carried out using two-step PCR with mutant oligonucleotides containing the specific substitutions. In the first step of the PCR the point mutations were introduced by using the mutant oligonucleotide and oligonucleotide catRylem 5'-GGCATC(EcoRI)GATTGCCTCCCAACTTTTAGTCTTG-3', which is complementary to nucleotides -84 to -113 relative to the transcriptional start site of the pheBA promoter. The first step of the PCR $\left(25\right.$ cycles) was: $1 \mathrm{~min}$ at $95^{\circ} \mathrm{C}, 1 \mathrm{~min}$ at $54{ }^{\circ} \mathrm{C}$, $1 \mathrm{~min}$ at $72{ }^{\circ} \mathrm{C}$. Then $2.5 \mathrm{U}$ Exol (Amersham) was added to the reaction mixture and the probes were incubated for $30 \mathrm{~min}$ at $37^{\circ} \mathrm{C}$ to remove the oligonucleotides used in the first step of the PCR. PCR products of $\sim 70 \mathrm{bp}$ were purified and used as oligonucleotides in the second step of the PCR with the oligonucleotide catRall 5'-GGCATC(ClaI)GATTGCCTCCCAACTTTTAGTCTTG-3', which is complementary to nucleotides +14 to +44 relative to the transcriptional start site of the pheBA promoter. The conditions of the second step of the PCR $\left(25\right.$ cycles) were: $1 \mathrm{~min}$ at $95^{\circ} \mathrm{C}, 3 \mathrm{~min}$ at $54{ }^{\circ} \mathrm{C}$, $1 \mathrm{~min}$ at $72^{\circ} \mathrm{C}$. The $158 \mathrm{bp}$ amplified DNA fragments were cloned using EcoRI and BamHI ends into pBluescript $\mathrm{SK}(+)$. All mutations were verified by dideoxy-sequencing with a Sequenase version 2.0 kit (Amersham). The pheBA promoter mutants were cloned into the promoter-probe vector $\mathrm{pKTlacZ}$ by using the Bam $\mathrm{HI}$ and $\mathrm{XhoI}$ ends.

$\boldsymbol{\beta}$-Galactosidase assay. Cells of $P$. putida PaW85 harbouring different $p h e B A$ promoter constructs were grown overnight to stationary phase. Sodium benzoate (final concentration $10 \mathrm{mM}$ ) was added to the growth medium to induce transcription from the pheBA promoter. The $\beta$-galactosidase $(\beta$ Gal) assay was performed as specified by Miller (1992). Protein concentration in crude lysates was measured by the method of Bradford (1976).

Overproduction of CatR and preparation of CatR lysate. The CatR protein was overexpressed in E. coli strain BL21(DE3) containing the expression plasmid pET11CatR (see above). Cells were grown in 1 litre $\mathrm{M} 9$ minimal medium at $20^{\circ} \mathrm{C}$. IPTG, final concentration $0.4 \mathrm{mM}$, was added at $\mathrm{OD}_{580} 0.5$, after which growth was allowed to continue for $4 \mathrm{~h}$. Cells were centrifuged at $4600 \mathrm{~g}$ for $10 \mathrm{~min}$ and suspended in $10 \mathrm{ml}$ CatR lysate buffer $(50 \mathrm{mM}$ Tris $/ \mathrm{HCl} \mathrm{pH} 7.5 ; 0.05 \mathrm{mM}$ EDTA; $10 \mathrm{mM} \mathrm{MgCl}$; $200 \mathrm{mM} \mathrm{NaCl} ; 1 \mathrm{mM} \mathrm{DTT} ; 0.05 \%$ Triton X-100; $10 \%, \mathrm{v} / \mathrm{v}$, glycerol). The cell suspension was sonicated and centrifuged at $25000 \mathrm{~g}$ for $10 \mathrm{~min}$.

Gel-mobility-shift assay. The primer CatRylem, labelled with $\left[\gamma_{-}{ }^{32} \mathrm{P}\right] \mathrm{dATP}$ using T4 polynucleotide kinase, and the primer CatRall were used for PCR amplification of the $158 \mathrm{bp}$ DNA fragments containing the wild-type or the mutant pheBA promoter region. The radiolabelled DNA fragments were purified by polyacrylamide gel electrophoresis. The CatR binding reaction was carried out in a volume of $20 \mu \mathrm{l}$. DNA probes $\left(200\right.$ c.p.m.) were incubated at $20^{\circ} \mathrm{C}$ for $20 \mathrm{~min}$ with CatR lysate $(0.001 \mathrm{mg}$ or $0.01 \mathrm{mg}), 1 \times$ binding buffer $(50 \mathrm{mM}$ Tris/HCl pH 7.5; $5 \mathrm{mM}$ EDTA; $250 \mathrm{mM} \mathrm{KCl;} 25 \mathrm{mM}$ $\mathrm{MgCl}_{2} ; 25 \mathrm{mM} \beta$-mercaptoethanol; $0.5 \%$ Triton X-100; $20 \%, \mathrm{v} / \mathrm{v}$, glycerol), $1 \mu \mathrm{g}$ bovine serum albumin, $5 \mu \mathrm{g}$ salmon sperm DNA and $100 \mu \mathrm{M}$ of the inducer CCM. After incubation, the reaction mixture was loaded on a pre-run $(20$ $\min ) 5 \%(\mathrm{w} / \mathrm{v})$ nondenaturing polyacrylamide gel. Electrophoresis was carried out at $3{ }^{\circ} \mathrm{C}$ in $0.5 \times$ Tris/borate/EDTA buffer, $\mathrm{pH} 7 \cdot 5$, at $10 \mathrm{~V} \mathrm{~cm}^{-1}$ for $2 \mathrm{~h}$. The gel was exposed to a phosphoimager screen.

Determination of the CatR binding activity of the pheBA promoter mutants. The gel-shift assays were performed three or four times in the presence and absence of $100 \mu \mathrm{M}$ CCM with the two different concentrations of CatR lysate (as indicated above) which yielded less than $100 \%$ bound radioactive probe for the wild-type pheBA promoter region. The bound DNA relative to the unbound DNA was quantified by Phosphoimager (Image Quant 4.2a software, Molecular Dynamics). The relative CatR binding activity for the pheBA promoter mutants was expressed as a percentage of the wildtype pheBA promoter binding.

\section{RESULTS}

\section{Site-directed mutagenesis of the pheBA promoter region bound by CatR}

The DNA sequences bound by CatR within the pheBA promoter region have been localized by DNase I footpinting (Parsek et al., 1995). In order to determine the critical nucleotides involved in DNA-protein interactions at the $p h e B A$ promoter, this DNA sequence was subjected to site-directed mutagenesis. Base substitutions were introduced into the RBS, the ABS and the 'hinge' region between the RBS and the ABS shown to be involved in DNA bending at the pheBA promoter (Parsek et al., 1995) (Fig. 2). The DNA fragments carrying the pheBA promoter with mutated RBS, ABS or 'hinge' regions were cloned into the promoter-probe vector pKTLacZ (Hõrak \& Kivisaar, 1998). The effect of the mutations on promoter activity was tested by measuring the $\beta$-Gal activity in cells of $P$. putida PaW85 grown in LB in the presence or absence of benzoate. CCM, the inducer of the pheBA operon, is an intermediate of the benzoate degradation pathway and is generated from it. Gel-shift assays were used to determine the effect of the mutations on the binding efficiency of CatR. The results of the study of the effect of the mutations on the pheBA promoter activity and CatR binding are shown in Table 1.

\section{Effect of mutations in the RBS region}

The mutations which were introduced into the RBS and changed the nucleotides in the interrupted inverted repeat ATACC-N ${ }_{5}$-GGTAT (see Fig. 2) negatively affected transcription from the $p h e B A$ promoter (Table 1). A drastic negative effect on $\beta$-Gal activity was observed in the case of the mutations 73AT, 72TG, 70CA, 69CA, 60AT and 59TG, and in the case of the double mutation $72 \mathrm{TC} / 73 \mathrm{AC}$ in both the presence and absence of benzoate. The mutation 63GA, which also changed the inverted repeat sequence, had a less marked effect on the pheBA promoter activity $(21 \%$ of the wildtype activity was retained in the presence of benzoate). The mutations $67 \mathrm{CA}$ and $66 \mathrm{CA}$ that were generated between the interrupted inverted repeat sequence exhibited almost wild-type levels of $\beta$-Gal activity in induced cells. The 74CT mutation lies upstream of the repeat sequence. However, it reduced the promoter 


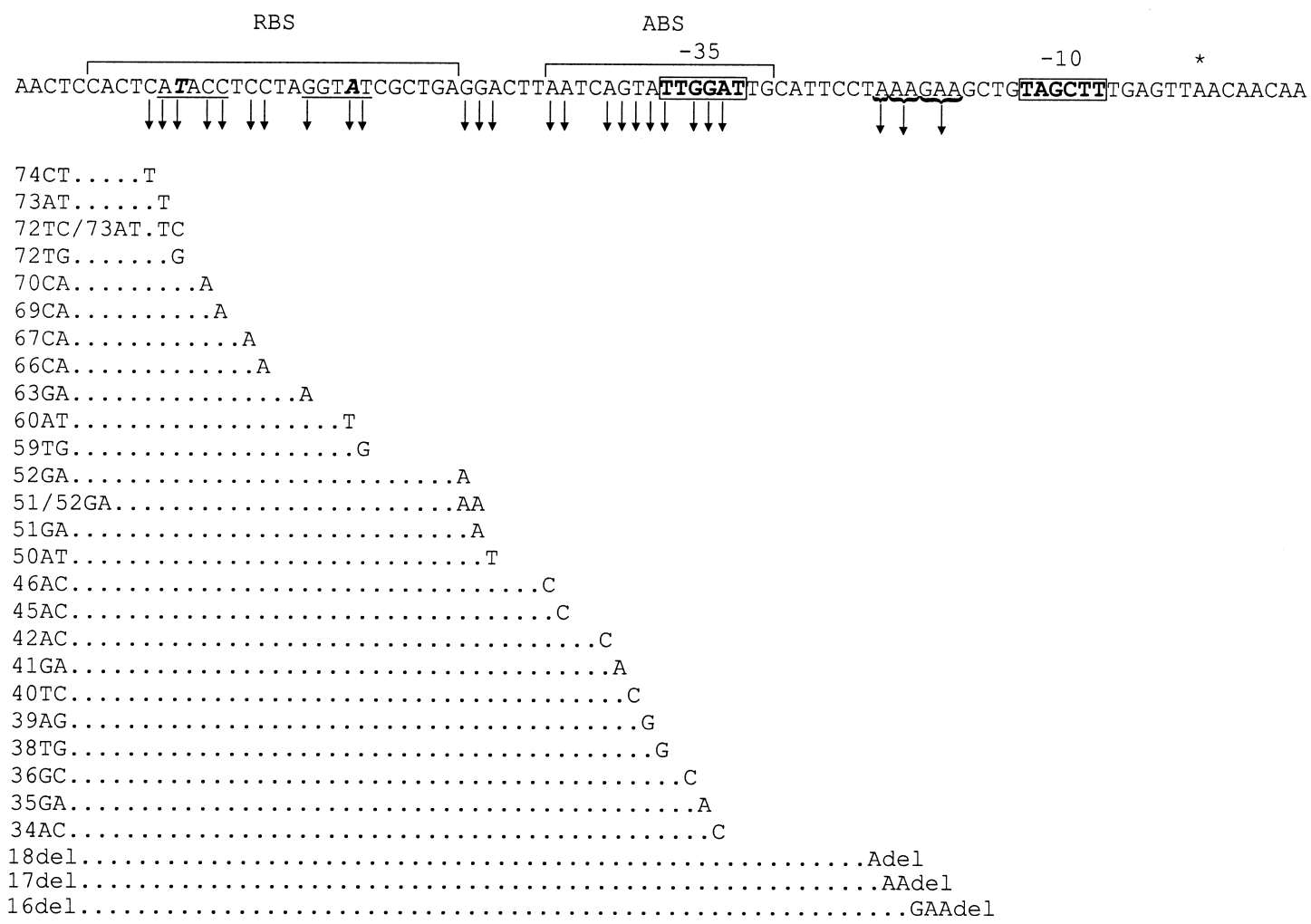

Fig. 2. Site-directed mutagenesis of the pheBA promoter region. The arrows mark the location of the mutations. The brackets indicate the location of the CatR-binding sites RBS and ABS. RBS and ABS are separated by the hinge region (not marked in the figure). The nucleotides of the interrupted inverted repeat are underlined and the boldface italic represents the $T$ and $A$ residues of the conserved $T-N_{11}$ - $A$ of the LysR binding motif. The nucleotides of the -35 and -10 elements of the pheBA promoter are in boldface and boxed. The deletion derivatives at positions -16 to -18 are referred to as del.

activity significantly in both the presence and absence of benzoate (only approximately $7 \%$ of the wild-type activity remained). The results of the gel-shift assay monitoring CatR binding to the RBS mutants were in good agreement with in vivo results: the mutations that negatively affected the expression of the pheBA also led to reduced CatR binding efficiency (Table 1 ).

\section{Effect of mutations in the 'hinge' region}

The CatR binding sites RBS and ABS are separated from each other by a 'hinge' region which has been postulated to be involved in DNA bending at the pheBA promoter (Parsek et al., 1995). Site-directed mutagenesis in the 'hinge' region, where the $G$ residues were substituted with A residues, was performed in order to generate a more flexible AT-rich region. The 52GA and 51GA mutations increased the efficiency of CatR binding in in vitro binding assays in the presence of CCM approximately $2 \cdot 5$ - and $3 \cdot 5$-fold, respectively, and this positive effect was also observed without the inducer (Table 1). A 2.5-fold increase in CatR binding efficiency was also observed in the case of the double mutation 51/52GA, which generated a stretch of four $\mathrm{A}$ residues in the 'hinge' region. However, the effect of these mutations in $\beta$-Gal assays was not so marked. Only the 52GA mutation demonstrated more than a twofold increase in expression of the pheBA promoter in the presence of benzoate; the level of expression of this mutant in the absence of inducer was enhanced $3 \cdot 5$-fold compared to the wild-type promoter (Table 1). The double mutation $51 / 52 \mathrm{GA}$ resulted in slightly elevated levels of expression and the 51GA mutation resulted in expression levels that were $80 \%$ of the wild-type levels in the presence of benzoate. Interestingly, the mutation $50 \mathrm{AT}$, which was neither expected to influence the flexibility of the hinge region nor was previously shown to be involved in the CatR binding on the basis of DNase I footprint analysis (Parsek et al., 1995), had a negative effect on pheBA promoter activity. This mutation also conferred a reduced CatR-binding efficiency, as observed in the gel-shift assay in the presence of CCM (Table 1).

\section{Effect of mutations in the ABS region}

The CatR ABS overlaps the -35 hexamers of the phe BA promoter and the $c a t B C A$ promoter. In the absence of CCM, CatR binds to the RBS in the catBCA promoter. Presence of CCM results in the occupation of an additional adjacent binding site, the ABS, and an approximately twofold increase in CatR's DNA binding 
Table 1. Effects of base substitutions in the pheBA promoter region on the CatRmediated in vivo activation and in vitro binding activity of the mutants

\begin{tabular}{|c|c|c|c|c|}
\hline \multirow[t]{2}{*}{ Mutant } & \multicolumn{2}{|c|}{ Activity and binding plus inducer } & \multicolumn{2}{|c|}{ Activity and binding minus inducer } \\
\hline & $\begin{array}{l}\text { In vivo activity of } \\
\text { phe } B A \text { promoter" }\end{array}$ & $\begin{array}{c}\text { Percentage } \\
\text { of wild-type } \\
\text { CatR } \\
\text { binding } \dagger\end{array}$ & $\begin{array}{l}\text { In vivo activity of } \\
\text { pheB } A \text { promoter* }\end{array}$ & $\begin{array}{c}\text { Percentage } \\
\text { of wild-type } \\
\text { CatR } \\
\text { binding } \dagger\end{array}$ \\
\hline Wild-type & $475 \cdot 7 \pm 55 \cdot 2(100)$ & 100 & $2 \cdot 6 \pm 0 \cdot 7(100)$ & 100 \\
\hline \multicolumn{5}{|l|}{ RBS } \\
\hline $74 \mathrm{CT}$ & $31 \cdot 7 \pm 9 \cdot 2(6 \cdot 7)$ & 25 & $0 \cdot 2 \pm 0 \cdot 05(7 \cdot 4)$ & 15 \\
\hline $73 \mathrm{AT}$ & $0 \cdot 12 \pm 0 \cdot 03(0 \cdot 6)$ & ND & $0 \cdot 13 \pm 0 \cdot 02(5 \cdot 2)$ & ND \\
\hline $72 \mathrm{TC} / 73 \mathrm{AC}$ & $2 \cdot 7 \pm 0 \cdot 3(0 \cdot 02)$ & ND & $0 \cdot 12 \pm 0 \cdot 01(4 \cdot 7)$ & ND \\
\hline $72 \mathrm{TG}$ & $11 \cdot 9 \pm 1 \cdot 5(2 \cdot 5)$ & ND & $0 \cdot 2 \pm 0 \cdot 02(8 \cdot 5)$ & ND \\
\hline $70 \mathrm{CA}$ & $5 \cdot 5 \pm 0 \cdot 4(1 \cdot 1)$ & $\mathrm{ND}$ & $0.2 \pm 0.04(8.5)$ & ND \\
\hline 69CA & $26 \cdot 7 \pm 2 \cdot 2(5 \cdot 6)$ & ND & $0 \cdot 3 \pm 0 \cdot 04(11)$ & ND \\
\hline $67 \mathrm{CA}$ & $451 \cdot 6 \pm 29 \cdot 5(95)$ & 115 & $1 \cdot 9 \pm 0 \cdot 2(75)$ & 130 \\
\hline $66 \mathrm{CA}$ & $410 \cdot 7 \pm 27 \cdot 5(86)$ & 150 & $1 \cdot 3 \pm 0 \cdot 3(52)$ & 140 \\
\hline 63GA & $99 \cdot 2 \pm 7 \cdot 5(21)$ & 40 & $0 \cdot 2 \pm 0 \cdot 02(6 \cdot 6)$ & 80 \\
\hline $60 \mathrm{AT}$ & $38 \cdot 2 \pm 4 \cdot 3(8)$ & ND & $0 \cdot 2 \pm 0 \cdot 02(9)$ & ND \\
\hline \multicolumn{5}{|l|}{ Hinge } \\
\hline $59 \mathrm{TG}$ & $15 \cdot 8 \pm 2 \cdot 9(3)$ & $\mathrm{ND}$ & $0 \cdot 2 \pm 0 \cdot 01(10)$ & ND \\
\hline $52 \mathrm{GA}$ & $1056 \cdot 5 \pm 154 \cdot 0$ & 280 & $9 \cdot 8 \pm 1 \cdot 4(375)$ & 120 \\
\hline $51 / 52 \mathrm{GA}$ & $637 \cdot 2 \pm 82 \cdot 6(134)$ & 260 & $2 \cdot 8 \pm 0 \cdot 6(107)$ & 130 \\
\hline $51 \mathrm{GA}$ & $378 \cdot 2 \pm 47 \cdot 4(80)$ & 370 & $2 \cdot 2 \pm 0 \cdot 1(84)$ & 150 \\
\hline $50 \mathrm{AT}$ & $56 \cdot 6 \pm 2 \cdot 2(12)$ & 40 & $0 \cdot 5 \pm 0 \cdot 1(20)$ & 105 \\
\hline \multicolumn{5}{|l|}{ ABS } \\
\hline $46 \mathrm{AC}$ & $0 \cdot 15 \pm 0 \cdot 01(0 \cdot 03)$ & 55 & $0 \cdot 17 \pm 0 \cdot 03(6 \cdot 8)$ & 90 \\
\hline $45 \mathrm{AC}$ & $0.13 \pm 0.03(0.03)$ & 60 & $0 \cdot 13 \pm 0.03(5 \cdot 2)$ & 135 \\
\hline $42 \mathrm{AC}$ & $0 \cdot 1 \pm 0.02(0.02)$ & 55 & $0 \cdot 15 \pm 0 \cdot 01(6)$ & 102 \\
\hline 41GA & $0.1 \pm 0.02(0.02)$ & 40 & $0 \cdot 17 \pm 0 \cdot 01(6 \cdot 5)$ & 135 \\
\hline $40 \mathrm{TC}$ & $16.4 \pm 1.5(3.5)$ & 50 & $0 \cdot 17 \pm 0.03(6 \cdot 8)$ & 120 \\
\hline $39 A G$ & $7 \cdot 0 \pm 1 \cdot 1(1 \cdot 5)$ & 50 & $0 \cdot 3 \pm 0 \cdot 05$ & 80 \\
\hline $38 \mathrm{TG}$ & $7 \cdot 2 \pm 0.8(1.5)$ & 75 & $0 \cdot 8 \pm 0 \cdot 1(31)$ & 130 \\
\hline $36 \mathrm{GC}$ & $9 \cdot 9 \pm 0.7(2)$ & 110 & $0 \cdot 2 \pm 0 \cdot 01(10)$ & 130 \\
\hline $35 \mathrm{GA}$ & $4 \cdot 9 \pm 1 \cdot 3(1)$ & 110 & $0 \cdot 2 \pm 0.03(8.5)$ & 120 \\
\hline $34 \mathrm{AC}$ & $277 \cdot 7 \pm 47 \cdot 6(58)$ & 75 & $0.7 \pm 0.06(26)$ & 110 \\
\hline
\end{tabular}

*Specific activity of $\beta$-Gal [nmol o-nitrophenol formed $\min ^{-1}(\mathrm{mg} \text { protein })^{-1}$ ] measured in $P$. putida PaW85 cells grown in the presence or absence of benzoate (inducer). Data (means \pm standard deviations) of at least five independent experiments are presented. The in vivo expression of the mutant promoters relative to the wild-type promoter $(\%)$ is shown in parentheses.

†In vitro CatR binding data expressed as the percentage of the wild-type binding activity in the presence or absence of CCM (inducer). ND, Not detectable.

affinity (Parsek et al., 1994, 1995). Binding of CatR to the ABS is cooperative and requires an intact RBS (Parsek et al., 1992, 1994). Thus, substituting the nucleotides necessary for the CatR binding to the ABS would result in a decrease in CatR's binding efficiency to the pheBA promoter region to half of that detected with the original promoter sequence in the presence of CCM. As expected, the base substitutions 39AG, 40TC, 41GA, $42 \mathrm{AC}$ and $46 \mathrm{AC}$ that were introduced upstream of the -35 element of the pheBA promoter led to approximately $50 \%$ CatR-binding efficiency to the pheBA promoter region in the presence of CCM as compared with the wild-type promoter (Table 1). In comparison to these substitutions, the effect of the mutations 45AC, $38 \mathrm{TG}$ and $34 \mathrm{AC}$ on CatR binding to the ABS was weaker $(60-75 \%$ of the CatR-binding efficiency to the wild-type promoter region was retained) and no negative effect was observed in the case of the mutations 36GC and 35GA (Table 1).

The in vivo study of the expression of the pheBA promoter carrying different mutations in the ABS region revealed a negative effect of all positions substituted. All mutations except $34 \mathrm{AC}$ led to greatly reduced levels of $\beta$ - 
Gal activity in the presence of benzoate (Table 1). The base substitutions in the ABS region also negatively affected the basal level of expression of the pheBA promoter (see the $\beta$-Gal activity in cells grown without benzoate in Table 1).

\section{Effect of the IBS on expression of the pheBA operon}

An additional binding site for CatR downstream of the catBCA promoter, called the internal binding site, IBS, has been identified (Chugani et al., 1998). The IBS negatively regulates the expression of the catBCA promoter. Occupation of the IBS by CatR was facilitated in the presence of the RBS and the ABS on the same DNA fragment, and the maintenance of phasing between the promoter and the IBS was important for the IBS-mediated repression (Chugani et al., 1998). On the basis of these data it was proposed that CatR bound to the DNA at the catBCA promoter, through formation of a DNA loop, could interact with CatR bound to the IBS, and that this interaction could cause impaired transcriptional activation from the catBCA promoter (Chugani et al., 1998). On the basis of DNase I footprint data a weak CatR-binding site downstream of the transcriptional start site of the pheBA operon $(+204$ to +221) was found as well (Parsek et al., 1996). In order to examine the effect of the pheBA IBS (see Fig. 1c) on the expression of the pheBA operon, this potential CatR binding site was deleted. The resulting construct, pIBS18del, contains an $18 \mathrm{bp}$ deletion of the IBS sequence and is replaced by an 8 bp foreign sequence. The 795 bp Eco47III-PstI fragment containing the pheBA promoter with the IBS sequence (designated as pIBS) and the deletion variant lacking this sequence were cloned into the plasmid pKTLacZ upstream of the promoterless lac $Z$ gene. The expression of the lac $Z$ transcriptional fusions in $P$. putida $\mathrm{PaW} 85$ grown in the presence of benzoate revealed an approximately twofold higher level of $\beta$-Gal activity in the case of the IBS deletion construct pIBS18del when compared with the original construct pIBS: $621 \pm 96$ versus $348 \pm 38 \mathrm{nmol}$ $\min ^{-1}$ (mg protein $)^{-1}$ (means $\pm \mathrm{SD}, n=5$ ). This indicates that the IBS of the pheBA operon could function as the cis-acting repressing element analogous to the IBS of the cat $B C A$ operon. However, the effect observed in this study was somewhat less (a twofold effect in comparison with the three- to fourfold effect found in the case of the catBCA system).

The IBS of the catBCA promoter closely matches the consensus sequence of the CatR-binding site RBS (Chugani et al., 1998). The IBS region of the pheBA operon contains the sequence ATACC at positions +207 to +211 , which is identical to one half of the interrupted inverted repeat of the RBS sequence (Fig. 1). The location of an A at position +220 (11 nt from the T of the sequence ATACC) matches the LysR-binding consensus $\mathrm{T}-\mathrm{N}_{11}$-A motif. We generated two mutations in the IBS region of the pheBA operon: the 208TC mutation, which substituted the T residue in the IBS to a $\mathrm{C}$ residue, and the 220A-GG mutation, which replaced the $\mathrm{A}$ residue at position +220 with two $\mathrm{G}$ residues (Fig. 1c). The effect of these mutations was tested using the pKTLacZ reporter system (constructs pIBS208TC and pIBS220A-GG, respectively) with cells grown in the presence of benzoate. Only a slight increase of the $\beta$-Gal activity was observed when the 208TC mutation was compared with the wild-type sequence: $397 \pm 35$ versus $348 \pm 38 \mathrm{nmol} \mathrm{min}^{-1}$ (mg protein $)^{-1}$. However, the $220 \bar{A}-G G$ mutation resulted in a twofold increase in the expression of the $\beta$-Gal activity [to $607 \pm 54 \mathrm{nmol} \mathrm{min}{ }^{-1}$ $\left.(\mathrm{mg} \text { protein })^{-1}\right]$ in comparison with the wild-type. The twofold positive effect of the IBS deletion and mutation 220A-GG was observed also in cells that were grown without the inducer (data not shown).

\section{Effect of the length of the spacer sequence between the -35 and -10 hexamers of the pheBA promoter on CatR-dependent transcriptional activation of the pheBA promoter}

The optimal distance between the -35 and -10 hexamers of the RNA polymerase $\sigma^{70}$-recognized promoters is usually $17 \mathrm{bp}$ (Stefano \& Gralla, 1982). The spacer sequence between the -35 and -10 hexamers of the pheBA promoter is abnormally long, $19 \mathrm{bp}$. The $19 \mathrm{bp}$ spacer adds an additional twist angle of at least $34^{\circ}$ and the two hexamers may be out of phase with respect to each other. This raised the question whether optimizing the distance between the -35 and -10 elements of the pheBA promoter could compensate for the requirement of CatR for transcriptional activation of this promoter. Using PCR, we made deletions in the spacer sequence of the pheBA promoter that reduced the distance between the hexamers from $19 \mathrm{bp}$ to 18,17 or $16 \mathrm{bp}$ and cloned the mutant promoters into the plasmid pKTLacZ (constructs pDEL18, pDEL17 and pDEL16, respectively; Fig. 2). Like cells with the wild-type promoter, the deletion mutants exhibited only a low basal level $\beta$-Gal activity both in the wild-type and in the CatR ${ }^{-}$background when bacteria were grown without the inducer (data not shown). When benzoate was added to the growth medium the $18 \mathrm{bp}$ spacer mutant showed a higher level of $\beta$-Gal activity than the control: $735 \pm 10$ versus $365 \pm 19 \mathrm{nmol} \mathrm{m^{-1 }}$ (mg protein $)^{-1}$. Reducing the distance between the -35 and -10 elements to $17 \mathrm{bp}$ or $16 \mathrm{bp}$ had a negative effect on transcriptional activation of the pheBA promoter: pDEL17 showed a twofold lower level of $\beta$-Gal activity $\left[184 \pm 20 \mathrm{nmol} \mathrm{min}{ }^{-1}(\mathrm{mg} \text { protein })^{-1}\right]$ than the construct carrying the wild-type promoter with the $19 \mathrm{bp}$ spacer, and pDEL16 demonstrated only the basal level of activity. Thus, optimizing the distance between the -35 and -10 hexamers of the pheBA promoter is not sufficient for CatR-independent transcriptional activation of this promoter.

\section{DISCUSSION}

The promoter of the plasmid-encoded phenoldegradative operon $p h e B A$ shows sequence similarity to the promoter of the chromosomal catechol-degradative catBCA genes in P. putida (Kasak et al., 1993). The chromosomal cat $R$ product positively regulates the 
expression of both promoters (Kasak et al., 1993; Parsek et al., 1995). Site-directed mutagenesis of the pheBA promoter region performed in this study revealed critical nucleotides involved in protein-DNA interactions at the pheBA promoter. Mutations in the interrupted inverted repeat in the RBS had drastic effects on CatR binding and activation of the pheBA promoter (Fig. 2, Table 1). Interestingly, base substitutions $67 \mathrm{GA}$ and $66 \mathrm{CA}$ between the repeat sequences did not have any effect. This indicates that the nucleotides in the interrupted inverted repeat may play an important role in the sequencespecific recognition of the RBS by CatR. Similar results were seen in a previous site-directed mutagenesis study of the catBCA promoter (Parsek et al., 1994). The mutation 74CT, which does not lie in the repeat, had a negative effect on the in vivo activity of the promoter and resulted in a lower CatR binding efficiency. Some mutations outside the inverted repeat of the catBCA promoter also had negative effects on the in vivo activity of the promoter (Parsek et al., 1994). The RBS sequence of the catBCA promoter contains the imperfect inverted repeat AGACC- $\mathrm{N}_{5}$-GGTAT. It harbours a G-N ${ }_{11}$-A motif instead of the consensus $\mathrm{T}-\mathrm{N}_{11}-\mathrm{A}$ of the LysRfamily binding motifs. The pheBA promoter has a perfect inverted repeat ATACC- ${ }_{5}$-GGTAT and CatR binds the $p h e B A$ promoter with greater affinity than the catBCA promoter region (Parsek et al., 1995). Changing the $\mathrm{G}$ to the consensus $\mathrm{T}$ in the catBCA RBS resulted in slightly elevated levels of the CatR binding and the promoter activation. However, the mutation 72TG, which made the repeat sequence of the pheBA RBS identical to that of the catBCA promoter, abolished CatR binding and only $2 \%$ of the wild-type promoter activity was observed under activating conditions. The possibility of different CatR-DNA interactions at the $\mathrm{RBS}$ of the pheBA and the catBCA promoter regions is also supported by the CatR-induced DNA bending data demonstrating a different degree of bending between the pheBA and the catBCA promoter regions in the absence of the inducer (Parsek et al., 1995). Moreover, the CatRbinding motif AGACC- $\mathrm{N}_{5}$-GGTAT is conserved between the RBS and the low-affinity binding site IBS of the catBCA promoter (Chugani et al., 1998). This finding also indicates that the conserved interrupted inverted repeat sequence is not the sole important feature for high-affinity binding of CatR.

Under activating conditions, in the presence of the inducer CCM, CatR binds to the pheBA promoter and the catBCA promoter as a tetramer. One dimer binds to the RBS and the second dimer binds cooperatively to the ABS (Parsek et al., 1994, 1995). The CatR binding site ABS encompasses the promoter -35 element. Since the CatR recognition elements are oriented on the opposite face of the DNA helix to the -35 element, both CatR and RNA polymerase may simultaneously interact with the same sequences from opposite sides of the DNA helix. Mutations in the ABS of the pheBA promoter fall into two groups on the basis of their effects: (1) mutations that affect both the promoter activity and CatR binding, and (2) mutations that affect negatively only the expression of the promoter (Fig. 2, Table 1). For example, the mutations $36 \mathrm{GC}$ and 35GA, which encompass the -35 hexamer sequence TTGGAT of the pheBA promoter, drastically reduced the level of promoter expression in both the presence and the absence of the inducer but did not affect CatR binding. This indicates that these two nucleotides may be involved in interaction of RNA polymerase with the promoter sequence. The -35 hexamer sequence TTGGAT of the pheBA promoter deviates from the -35 consensus sequence TTGACA at three positions. Therefore, it was unexpected that changing the nucleotide from $G$ to $A$ at position -35 relative to the pheBA transcriptional start (mutation 35GA) would inactivate the promoter, since such a change made the promoter sequence similar to the $\sigma^{70}$-recognized promoter consensus. Comparison of the sequences of the -35 elements of the pheBA promoter and the catBCA promoter reveals that they are highly conserved (sequences TTGGAT and TTGGAC, respectively). The -35 hexamer of the promoter of the chlorocatechol-degradative genes $c l c A B D$ is identical to that of the catBCA promoter and it was shown that CatR and ClcR activate transcription via a conserved mechanism (McFall et al., 1997). The elimination of expression from the pheBA promoter as a result of the 35GA mutation and the conservation of a $G$ nucleotide instead of the consensus nucleotide $\mathrm{A}$ in these three promoters indicate that this $\mathrm{G}$ nucleotide is important for RNA polymerase interactions with the CatR-and ClcR-regulated promoters.

A 19 bp spacer separates the -35 and -10 hexamers of the pheBA promoter and the catBCA promoter. The promoter of the mercury-resistance operon mer also deviates from the $\sigma^{70}$-recognized promoter consensus in that there is a $19 \mathrm{bp}$ spacer region separating the two hexamers of the promoter (Parkhill \& Brown, 1990). Mercury-dependent activation of this promoter brings the -10 and -35 elements into a better helical alignment through a MerR-mediated untwisting effect at the spacer DNA (Ansari et al., 1992; O'Halloran et al., 1989). Single and double base-pair deletions in the interhexamer spacer of the mer operon promoter resulted in MerR-independent transcriptional activation of this promoter (Parkhill \& Brown, 1990). In the case of the promoter of the mom gene (encoding a DNAmodification function of the $\mathrm{Mu}$ phage), the $19 \mathrm{bp}$ suboptimal spacer region is also known to function (Bolker et al., 1989). C-protein-mediated torsional changes reorient the -10 and -35 elements to a favourable conformation for RNA polymerase binding at this promoter (Basak \& Nagaraja, 1998). The deletions made by us to optimize the length of the spacer region of the pheBA promoter did not compensate for CCM-dependent CatR-mediated activation. However, these data show that the exact orientation of the CatRand RNA-polymerase-binding elements on the DNA helical face is important. Deletion of $2 \mathrm{bp}$ or more from the spacer sequence had a negative influence on the promoter activity: the deletion variant with the $16 \mathrm{bp}$ spacer was not activated at all. 
CatR induces a DNA bend in the 'hinge' region between the CatR-binding sites RBS and ABS of the pheBA and catBCA promoters (Parsek et al., 1995). In the presence of CCM, the DNA bending angle of the catBCA promoter is partially relaxed. In the case of the pheBA promoter, CatR bends the DNA in the presence or absence of inducer to the relaxed bending angle of the catBCA promoter when CCM is present. Although the fixed DNA bending angle may be important for the CatR-mediated transcriptional activation of both the promoters, some other inducer-dependent CatRmediated conformational changes at the promoterRNA polymerase complex are required as well. The requirement of the carboxy-terminal domain of the $\alpha$ subunit $(\alpha-\mathrm{CTD})$ of RNA polymerase was demonstrated for the activation of the $p h e B A$ and the catBCA promoters (Chugani et al., 1997). The pattern of activation of these promoters resembles the pattern of activation for upstream enhancer element (UP element)dependent activation more closely than cyclic AMP receptor protein (CRP)-dependent activation (Murakami et al., 1996). Therefore, it was suggested that the $\alpha$-CTD might interact directly not only with CatR but also with the DNA at the putative UP element (Chugani et al., 1997).

The substitution of an A nucleotide with a $\mathrm{G}$ nucleotide (52AG mutation) in the catBCA promoter 'hinge' region lowered the activation level of the promoter approximately fivefold but did not affect CatR binding (Parsek et al., 1994). It was thought that this change would alter the flexibility of the 'hinge' region of the promoter (Parsek et al., 1994, 1995). The in vivo and in vitro effects of the base substitutions that were made in the 'hinge' region of the pheBA promoter were somewhat unexpected (Table 1). The binding efficiency of CatR as determined by the gel-shift assay was increased approximately threefold by the substitution of $G$ nucleotides with A nucleotides. Concurrently, the increase in the in vivo expression of the promoter (twofold) was seen only in one particular case (mutation 52GA). The reason for such a discrepancy is unclear. According to the model presented by Chugani et al. (1997, 1998), both CatR and RNA polymerase may simultaneously interact with the same sequences from opposite sides of the helix and the $\alpha$-CTD most likely interacts with the nucleotides located between the RBS and ABS. Therefore, it is tempting to speculate that the mutation 52GA improved the binding of $\alpha$-CTD to the putative UP element sequence of the pheBA promoter for its activation, thereby enhancing the level of transcriptional activation from the promoter.

The third CatR binding site, IBS, was localized within the catB structural gene (Chugani et al., 1998). The cooperativity observed in DNase I protection studies and the phasing dependence of IBS function indicated that the CatR dimers, bound to the RBS and ABS, interact with the CatR molecules bound to the IBS, with the looping out of the intervening DNA (Chugani et al., 1998). This interaction resulted in reduced transcriptional activation from the catBCA promoter. The presence of the CatR low-affinity binding site has also been suggested in the case of the pheBA operon at positions +204 to +221 with respect to the transcriptional start site of the operon (Parsek et al., 1996). In this study we have examined the possible biological effect of this site on the regulation of the pheBA operon. A study of the effect of base substitutions in the IBS region and the deletion of this site indicated that the IBS of the pheBA operon affects transcription from the pheBA promoter in a similar manner as in the case of IBS-mediated repression of the catBCA promoter.

The genetic organization of the pheBA operon is unusual: besides IS1411, which flanks the pheBA genes downstream, a second IS element, IS1472, is located between these genes and their promoter (Fig. 1a). A very similar IS element, IS1384, which shares approximately 99\% homology at the DNA level to IS1472, has been found in phenol-degrading Pseudomonas sp. strain $\mathrm{H}$ isolated from soil in Germany (GenBank accession number AF052751). The IBS of the pheBA operon is located between the left IR and the transposase gene tnpA of the IS element IS1472 (Fig. 1c). A sequence identical to the IBS of the pheBA operon is also present in IS1384. Thus, the location of the IBS of the pheBA operon in IS1472 demonstrates that, in principle, any sequence could play a role of IBS if this sequence can function as a binding site for CatR and has an appropriate location relative to the CatR-regulated promoter. It is also a good example of how mobile DNA elements can modulate the expression of neighbouring genes.

Comparative studies of the interaction of CatR at the promoters of the pheBA and catBCA operons have revealed that the CatR-mediated activation mechanism is well conserved despite the different origins of these operons. The pheBA gene cluster appears to be flanked by transposable elements (Kasak et al., 1993; Kallastu et al., 1998). This in turn could allow a rapid movement of these genes from one DNA molecule to another. After the release of the laboratory $P$. putida strain carrying the pheBA genes on a plasmid into a phenol-contaminated mining area in Estonia, horizontal transfer of the pheBA operon and its expression in different soil bacteria was observed (Peters et al., 1997). In all isolates degrading phenol via the ortho pathway and harbouring the phe $B A$ genes integrated into other plasmids, the original pheBA promoter sequence was present as before. Thus, the universal mechanism of CatR-mediated transcriptional activation could be of great importance in enabling catechol-degrading soil bacteria to expand their substrate range via horizontal transfer of the phenoldegradation genes without the need for subsequent extensive genetic rearrangements.

\section{ACKNOWLEDGEMENTS}

This work was supported by NIH Fogarty International Research Collaboration Award (Grant RO3 TW00490-01) and in part by grant 2323 from the Estonian Science Foundation. Work in the Chakrabarty lab was supported by PHS grant ES04050-13 from the National Institute of En- 
vironmental Health Sciences (NIEHS). We thank R. Hõrak for help in construction of some pheBA promoter mutants.

\section{REFERENCES}

Adams, M. H. (1959). Bacteriophages, pp. 445-447. New York: Interscience Publishers.

Aldrich, T. L. \& Chakrabarty, A. M. (1988). Transcriptional regulation, nucleotide sequence, and localization of the promoter of the catBC operon in Pseudomonas putida. J Bacteriol 170, 1297-1304.

Ansari, A. Z., Chael, M. L. \& O'Halloran, T. V. (1992). Allosteric underwinding of DNA is a critical step in positive control of transcription by Hg-MerR. Nature 355, 87-89.

Basak, S. \& Nagaraja, V. (1998). Transcriptional activator C protein-mediated unwinding of DNA as a possible mechanism for mom gene activation. J Mol Biol 284, 893-902.

Bayley, S. A., Duggleby, C. J., Worsey, M. J., Williams, P. A., Hardy, K. G. \& Broda, P. (1977). Two modes of loss of the TOL function from Pseudomonas putida mt-2. Mol Gen Genet 154, 203-204.

Bolker, M., Wulczyn, F. G. \& Kahmann, R. (1989). Role of bacteriophage $\mathrm{Mu} \mathrm{C}$ protein in activation of the mom gene promoter. J Bacteriol 171, 2019-2027.

Bradford, M. M. (1976). A rapid and sensitive method for the quantitation of microgram quantities of protein utilizing the principle of protein-dye binding. Anal Biochem 72, 248-254.

Carter, P., Bedoulle, H. \& Winter, G. (1985). Improved oligonucleotide site-directed mutagenesis using M13 vectors. Nucleic Acids Res 13, 4431-4443.

Chugani, S. A., Parsek, M. R., Hershberger, C. D., Murakami, K., Ishihama, A. \& Chakrabarty, A. M. (1997). Activation of the catBCA promoter: probing the interaction of CatR and RNA polymerase through in vitro transcription. J Bacteriol 179, 2221-2227.

Chugani, S. A., Parsek, M. R. \& Chakrabarty, A. M. (1998). Transcriptional repression mediated by LysR-type regulator CatR bound at multiple binding sites. J Bacteriol 180, 2367-2372.

Coco, W. M., Rothmel, R. K., Henikoff, S. \& Chakrabarty, A. M. (1993). Nucleotide sequence and initial functional characterization of the $c l c R$ gene encoding a LysR family activator of the clcABD operon in Pseudomonas putida. J Bacteriol 175, 417-427.

Franz, B. \& Chakrabarty, A. M. (1987). Organization and nucleotide sequence determination of a gene cluster involved in 3chlorocatechol degradation. Proc Natl Acad Sci USA 84, 4460-4464.

Goethals, K., Van Montagu, M. \& Holsters, M. (1992). Conserved motifs in a divergent nod box of Azorhizobium caulinodans ORS571 reveal a common structure in promoters regulated by LysR-type proteins. Proc Natl Acad Sci USA 89, 1646-1650.

Hanahan, D. (1983). Studies on the transformation of E. coli with plasmids. J Mol Biol 166, 577-580.

Harayama, S. \& Timmis, K. N. (1989). Catabolism of aromatic hydrocarbons by Pseudomonas. In Genetics of Bacterial Diversity, pp. 151-174. Edited by D. A. Hopwood \& K. E. Chater. London: Academic Press.

Herrmann, H., Janke, D., Kejsna, S. \& Roy, M. (1988). In vivo generation of R68.45-pPGH1 hybrid plasmids conferring a $\mathrm{Phl}^{+}$ (meta-pathway) phenotype. Mol Gen Genet 214, 173-176.

Hõrak, R. \& Kivisaar, M. (1998). Expression of the transposase gene $\operatorname{tnp} A$ of $\mathrm{Tn} 4652$ is positively affected by integration host factor. J Bacteriol 180, 2822-2829.
Kallastu, A., Hõrak, R. \& Kivisaar, M. (1998). Identification and characterization of IS1411, a new insertion sequence which causes transcriptional activation of the phenol degradation genes in Pseudomonas putida. J Bacteriol 180, 5306-5312.

Kasak, L., Hõrak, R., Nurk, A., Talvik, K. \& Kivisaar, M. (1993). Regulation of the catechol 1,2-dioxygenase- and phenol monooxygenase-encoding pheBA operon in Pseudomonas putida PaW85. J Bacteriol 175, 8038-8042.

Kivisaar, M., Hõrak, R., Kasak, L., Heinaru, A. \& Habicht, J. (1990). Selection of independent plasmids determining phenol degradation in Pseudomonas putida and the cloning and expression of genes encoding phenol monooxygenase and catechol 1,2dioxygenase. Plasmid 24, 25-36.

Kukor, J. J. \& Olsen, R. H. (1990). Molecular cloning, characterization, and regulation of a Pseudomonas pickettii PKO1 gene encoding phenol hydroxylase and expression of the gene in Pseudomonas aeruginosa PAO1. J Bacteriol 172, 4624-4630.

McFall, S. M., Klem, T. J., Fujita, N., Ishihama, A. \& Chakrabarty, A. M. (1997). DNase I footprinting, DNA bending and in vitro transcription analyses of ClcR and CatR on the $c l c A B D$ promoter: evidence of a conserved transcriptional activation mechanism. Mol Microbiol 24, 965-976.

McFall, S. M., Chugani, S. A. \& Chakrabarty, A. M. (1998). Transcriptional activation of the catechol and chlorocatechol operons: variations on a theme. Gene 223, 257-267.

van der Meer, J. R., Frijters, A. C. J., Leveau, J. H. J., Eggen, R. I. L., . Zehnder, A. J. B. \& de Vos, W. M. (1991). Characterization of the Pseudomonas sp. strain P51 gene $t c b R$, a LysR-type transcriptional activator of the $t c b C D E F$ chlorocatechol oxidative operon, and analysis of the regulatory region. J Bacteriol 173, 3700-3708.

Miller, J. H. (1992). A Short Course in Bacterial Genetics. Cold Spring Harbor, NY: Cold Spring Harbor Laboratory.

Murakami, K., Fujita, N. \& Ishihama, A. (1996). Transcription factor recognition surface on the RNA polymerase $\alpha$ subunit is involved in contact with the DNA enhancer element. EMBO J 15, $4358-4367$.

O’Halloran, T. V., Frantz, B., Shin, M. K., Ralston, D. M. \& Wright, J. G. (1989). The MerR heavy metal receptor mediates positive activation in a topologically novel transcription complex. Cell $\mathbf{5 6}$, 119-129.

Ornston, L. N. (1966). The conversion of catechol and protocatechuate to $\beta$-ketoadipate by Pseudomonas putida. IV. Regulation. J Biol Chem 241, 3800-3810.

Parkhill, J. \& Brown, N. L. (1990). Site-specific insertion and deletion mutants in the mer promoter-operator region of Tn501; the nineteen base-pair spacer is essential for normal induction of the promoter by MerR. Nucleic Acids Res 18, 5157-5162.

Parsek, M. R., Shinabarger, D. L., Rothmel, R. K. \& Chakrabarty, A. M. (1992). Role of CatR and cis,cis-muconate in the activation of the catBC operon involved on benzoate degradation in Pseudomonas putida. J Bacteriol 174, 7798-7806.

Parsek, M. R., Ye, R. W., Pun, P. \& Chakrabarty, A. M. (1994). Critical nucleotides in the interaction of a LysR-type regulator with its target promoter region. J Biol Chem 269, 11279-11284.

Parsek, M. R., Kivisaar, M. \& Chakrabarty, A. M. (1995). Differental DNA bending introduced by the Pseudomonas putida LysR-type regulator, CatR, at the plasmid-borne $p h e B A$ and chromosomal catBC promoters. Mol Microbiol 15, 819-828.

Parsek, M. R., McFall, S. M. \& Chakrabarty, A. M. (1996). Evolution of regulatory systems of biodegradative pathways. In Molecular Biology of Pseudomonads, pp. 135-152. Edited by T. 
Nakazawa, K. Furukawa, D. Haas \& S. Silver. Washington, DC: American Society for Microbiology.

Peters, M., Heinaru, E., Talpsep, E., Wand, H., Stottmeister, U., Heinaru, A. \& Nurk, A. (1997). Acquisition of a deliberately introduced phenol degradation operon, pheBA, by different indigenous Pseudomonas species. Appl Environ Microbiol 63, 4899-4906.

Rothmel, R. K., Aldrich, T. L., Houghton, J. E., Coco, W. M., Ornston, L. N. \& Chakrabarty, A. M. (1990). Nucleotide sequencing of Pseudomonas putida catR: positive regulator of the cat $B C$ operon is a member of LysR family. J Bacteriol 172, 922-931.

Rothmel, R. K., Shinabarger, D. L., Parsek, M. R., Aldrich, T. L. \& Chakrabarty, A. M. (1991). Functional analysis of the Pseudomonas putida regulatory protein CatR: transcriptional studies and determination of the CatR DNA-binding site by hydroxylradical footprinting. J Bacteriol 173, 4717-4724.

Sharma, R. C. \& Schimke, R. T. (1996). Preparation of electrocompetent E. coli using salt-free growth medium. BioTechniques 20, 42-44.
Shingler, V., Powlowski, J. \& Marklund, U. (1992). Nucleotide sequence and functional analysis of the complete phenol/3,4dimethylphenol catabolic pathway of Pseudomonas sp. Strain CF600. J Bacteriol 174, 711-724.

Stefano, J. E. \& Gralla, J. D. (1982). Spacer mutations in the lac ps promoter. Proc Natl Acad Sci USA 79, 1069-1072.

Studier, F. W. \& Moffatt, B. A. (1986). Use of bacteriophage T7 polymerase to direct selective high-level expression of cloned genes. J Mol Biol 189, 113-130.

Tsuda, M., Minegishi, K.-I. \& lino, T. (1989). Toluene transposons Tn4651 and Tn4653 are class II transposons. J Bacteriol 171, $1386-1363$.

Wigmore, G. J., DiBerardino, D. \& Bayly, R. C. (1977). Regulation of the enzymes of the meta-cleavage pathway of Pseudomonas putida: a regulatory model. J Gen Microbiol 100, 81-87.

Received 2 July 1999; revised 24 September 1999; accepted 4 October 1999 